\title{
The MUNI Photometric Archive
}

\author{
Marek Chrastina, Miloslav Zejda, and Zdeněk Mikulášek
}

Department of Theoretical Physics and Astrophysics, Faculty of Science, Masaryk University, Kotlářská 2, CZ-611 37 Brno, Czech Republic, homepage: http://astro.physics.muni.cz

\begin{abstract}
In the 1990s of the last century, CCD cameras became more reachable. Due to many advantages of CCD cameras, astronomers began using them as the primary detector for photometry of stellar objects. A typical observatory, which operates one telescope at a time, obtained $0.5 \mathrm{~TB}$ of raw data during two decades, that means one million about $500 \mathrm{kB}$-sized files. There are several observatories in the Czech Republic and Slovakia (taking into account all scientific, public as well as private ones). A rough estimate of the total amount of this photometric data is $10 \mathrm{~TB}$, which could be a very interesting source of observational data. Unfortunately, these data are not available online. These data are stored in observatory archives in arbitrary format. Often it is not even possible to find requested data. We have decided to change this state by establishing a common archive of raw photometric data, which would be available online together with tools for searching, listing etc. We already defined the data format, file and directory structure of our archive. We developed sophisticated tools for archive maintenance as well. Our goal is to provide data storage with simple and straightforward access and we are ready to interconnect with the VO right after the IVOA Photometry Data Model will be released.
\end{abstract}

Keywords. astronomical data bases, techniques: photometric, methods: data analysis

\section{Introduction}

CCD photometry was initiated at Masaryk University Observatory in 1996, when the main $0.62 \mathrm{~m}$ reflector was equipped with an SBIG ST-8 camera. Most of the observations were focused on variable star measurements. However, observations of comets, minor planets, extrasolar planets, star clusters and GRB afterglows were also obtained. All data were stored in FITS files on hard disks, CDs and DVDs. We obtained approximately $0.5 \mathrm{~TB}$ raw photometric data (including calibration images) during 15 years of camera operation. In other words, we have circa one million about $500 \mathrm{kB}$-sized files in our archive. Unfortunately, the data are not located in any kind of database, therefore they are without any online access.

There are several observatories in Czech Republic and Slovakia (taking into account all scientific, public as well as private ones). Unfortunately, the situation with their raw data is more or less the same, that means no database, no online access. These data are stored in observatory archives in arbitrary format. Often it is not even possible to find requested data.

Our rough estimate of the total amount of photometric data obtained in our region is about 10 TB. Just for comparison, SDSS DR7 contains 15.7 TB of images [e1]. Each observatory has a different scientific programme, but each of them worked over quite a long timeline. Often they provide long-term monitoring of several objects. In addition, the CCD provides measurements of the whole sky field, thus we can take advantage of the field overlapping. The field can also contain objects, that may be interesting for someone else. 


\section{MUNI Photometric Archive}

The MUNI Archive can be a very interesting source of observational data. Data are not available online from a single location. That is the problem which prevents further usage of the data. For that reason, we have decided to establish a single common archive of raw photometric data.

Due to the arbitrary format of a particular data source, we have to convert data to one specific uniform data format. We used restricted alternation of standard FITS format. We proposed an explicitly defined set of keywords. Furthermore, we propose a strict naming convention for FITS-file names and directory structure. There are many FITS files, thus we have developed a package of useful scripts for converting FITS files into the uniform format and structure (Chrastina et al. 2010).

\section{Acknowledgements}

This work has been supported by grants MUNI/A/0968/2009, GAAV IAA301630901 and MEB051018. Thanks also to Gabriel Szász and Filip Hroch for their cooperation.

\section{References}

e1, SDSS DR7 (The Sloan Digital Sky Survey Data Release 7), http://www.sdss.org/dr7/ Chrastina, M., Zejda, M., \& Mikulášek, Z. 2010, in "Binaries Key to Comprehension of the Universe," eds. A. Prsa et al., ASP-CS, 435, 83 